\title{
China: nuevos desafíos en la relación entre educación y mejora en la calidad de vida
}

\section{China: New Challenges in the Relationship between Education and Improvement in the Quality of Life}

Gan Yutian ${ }^{1}$

\section{Resumen:}

En este trabajo se analiza el rol de la educación como factor fundamental para la mejora de la calidad de vida de la población. Si bien, no es el único elemento que influye en este proceso, sí es una variable de jerarquía mayor.

En los últimos 40 años, China ha emprendido reformas educativas profundas; en consecuencia, la estabilidad económica y la reducción de la pobreza en algunas regiones de este país ha sido constante. Una de las razones de esta tendencia ha sido el desarrollo de políticas educativas a largo plazo, que han contribuido a consolidar el crecimiento económico.

Por tanto, el análisis de la educación, a partir de las políticas ejercidas por el Estado, podrá recrear un panorama más profundo acerca de los factores que han influido en el desarrollo de China en los últimos años.

Palabras clave: China, Educación, Desarrollo económico, Calidad de vida.

\begin{abstract}
:
This paper analyzes the role of education as a main factor for improving the people's quality of life. Although, it is not the only element that influences this

\footnotetext{
1 Profesora de la Universidad de Changzhou, China, e investigadora del Centro de Estudios Latinoamericanos (CELA) de la Universidad de Changzhou.
} 
process, it is a fundamental variable.

In the last 40 years, China has undertaken profound educational reforms; consequently, economic stability and the reduction of poverty in some regions of this country has been constant. One of the reasons for this trend has been the development of long-term educational policies that have helped to consolidate economic growth.

Therefore, the analysis of education, based on the policies exerted by the government, will be able to recreate a deeper panorama about the factors that have influenced the development of China in recent years.

Keywords: China, Education, Economic Development, People's Quality of Life.

\section{Introducción}

Desde las reformas realizadas en China a partir de 1978, la educación, la ciencia y la tecnología han sido factores clave de las políticas del gobierno; en particular, la educación entendida en todos sus niveles y con énfasis en el nivel superior para generar más valor agregado a la sociedad y mayores oportunidades para sus miembros. Este enfoque se ve claramente reflejado en el crecimiento y el desarrollo económico que ha experimentado el país desde esa época.

Si bien, la educación no es el único factor que genera desarrollo en las sociedades, es un factor fundamental con el que se sustentan muchas de las políticas del Estado. La educación es una herramienta individual y social que sienta las bases para el desarrollo.

Según Sachs (2017), las causas de la pobreza extrema están habitualmente relacionadas con múltiples factores. Destacan las carencias en:

1) Capital humano: salud, nutrición y capacitación.

2) Capital industrial: maquinarias, instalaciones y transporte motorizado.

3) Capital de infraestructura: carreteras, energía, agua y aeropuertos.

4) Capital natural: tierra cultivable, biodiversidad y ecosistemas. 
5) Capital institucional público: legislación, sistema judicial y políticas públicas.

6) Capital intelectual: saber práctico, científico y tecnológico.

De acuerdo con el autor, la educación es un elemento fundamental para la reducción de la pobreza y, en consecuencia, para mejorar la calidad de vida de la población.

\section{Calidad de vida de la población}

La calidad de vida, vista como una expresión del desarrollo, contempla elementos de naturaleza cualitativa que dificultan su medición. Además, existe un problema en cuanto a los indicadores que tienen que ver con la definición de este concepto.

Existen diferentes autores que trabajan este tema; para Dennis, Williams, Giangreco y Cloninger (1993), los enfoques de investigación sobre calidad de vida son variados, pero podrían acotarse en dos tipos:

a) Los enfoques cuantitativos, cuyo propósito es operacionalizar la calidad de vida. Para ello se han estudiado diferentes indicadores: sociales -se refieren a condiciones externas relacionadas con el entorno, como la salud, el bienestar social, la amistad, el estándar de vida, la educación, la seguridad pública, el ocio, el vecindario, la vivienda, etc.-; psicológicos -miden las reacciones subjetivas del individuo a la presencia o ausencia de determinadas experiencias vitales-, y ecológicos -miden el ajuste entre los recursos del sujeto y las demandas del ambiente.

b) Los enfoques cualitativos, cuyo método es escuchar al sujeto de estudio, mientras relata sus experiencias, desafíos y problemas. Intentan ubicar las mejores estrategias para que los servicios sociales pueden apoyar eficazmente a los individuos.

Hay varios indicadores para medir la calidad de vida de la población, pero hay que entenderla como un proceso dinámico, en el que una persona o familia 
determinada cambia constantemente y de forma casi imperceptible. Por lo anterior, se puede hablar de un "x" nivel de calidad de vida en un momento o periodo específico; en tanto, existen seis fuentes de bienestar o de posible medición:

1.- El ingreso corriente.

2.- Los derechos de acceso a los servicios o bienes gubernamentales (gratuitos o de bajo costo).

3.- La propiedad (que conforma el patrimonio básico).

4.- Los niveles educativos, las habilidades y las destrezas, asumidos como expresiones de la capacidad de entender y actuar en el mundo social.

5.- El tiempo disponible para la educación formal o informal, la recreación, el descanso y las tareas domésticas.

6.- La propiedad de activos no básicos como un automóvil y una vivienda, y la capacidad de endeudamiento del individuo y del hogar.

Por otra parte, la población tiene distintas capacidades de respuesta al riesgo; esta capacidad es menor en el caso de quienes viven en condición de pobreza, y es mayor -aunque precaria- entre quienes son vulnerables desde el punto de vista socioeconómico.

Para los hogares que han salido hace poco tiempo de una situación de pobreza o cuyos ingresos los ubican de forma leve sobre la línea de pobreza, un evento crítico puede determinar su recaída. Estos hogares son, por lo tanto, "no pobres", pero vulnerables al empobrecimiento. Su situación es diferente a la de los hogares "no pobres autónomos", que son menos vulnerables tanto por contar con mayores activos y/o ingresos como por tener un mejor acceso a esquemas de aseguramiento de tipo contributivo, y distinta a la de los "pobres", que pueden ser empujados hacia la indigencia o bien, hacia la pobreza crónica si se encuentran en situación de pobreza transicional. (Cecchini y Martínez, 2011)

Asimismo, hay diferencias entre los "pobres crónicos" y los "pobres transicionales", que dependen del tiempo en que los individuos han experimentado una privación significativa de sus capacidades y de su movilidad, en torno a la línea de pobreza. (Hulme \& Shepherd, 2003) 


\subsection{El índice de desarrollo humano}

El índice de desarrollo humano(IDH) es un indicador elaborado por las Naciones Unidas para medir el progreso de un país. A diferencia de los indicadores anteriores que medían el desarrollo económico de un país, el IDH analiza la salud, la educación y los ingresos. Cuanto mayor es el índice, mejor estará situado el país en el ranking internacional. A continuación se describen los factores evaluados:

- Salud (esperanza de vida al nacer). Se mide en el IDH utilizando un valor mínimo de 20 años y un valor máximo de 83.57. De forma que, por ejemplo, el componente de longevidad para un país cuya esperanza de vida al nacer sea de 55 años, será de 0,551.

- Educación. Se mide a través de los años de escolarización contemplados para adultos y los años de escolarización previstos para niños y niñas en edad escolar.

- Componente de riqueza (o estandar de vida digna). Se mide a través del ingreso nacional brutor (INB) per cápita (\$PPP), en lugar del producto interno bruto (PIB) per cápita (\$PP). Los límites de mínimo y máximo son $100 \$(P P P)$ y $87,478 \$(P P P)$.

\section{Educación como factor para la mejora de la calidad de vida}

La educación es una de las variables fundamentales para el desarrollo de las personas y el acceso a la igualdad de oportunidades; en ese sentido, contribuye al desarrollo económico. Como afirma Mosquera (2010), la educación -entendida como un proceso gradual de adquisición de conocimientos y habilidades- permite potencializar las capacidades del individuo y transformarlo en un agente productivo, en la medida en que favorece al valor agregado y mejora su entorno.

La meta de la educación es la formación holística de seres para el trabajo y la convivencia social, por lo que no basta con que se impartan conocimientos, sino que se deben incorporar valores que la legitimen como protagonista de la 
transformación social. Asimismo, considerando que la educación se constituye como un aspecto fundamental dentro del conjunto de factores sociales vinculados al desarrollo técnico-social, debe ser incluida dentro de toda estrategia de desarrollo de las naciones como punto absolutamente necesario en la actual sociedad del conocimiento. En ésta última se toma como factor determinante del aumento en el desarrollo de los pueblos y, en consecuencia, de los elementos considerados representativos de la calidad de vida. Así pues, existe una relación innegable e indestructible entre el nivel educativo-cultural de los pueblos, su desarrollo económico y su calidad de vida. (Magallanes, 2012)

Schultz (1961) y Denison (1962) mostraron que la parte del crecimiento no explicada por los factores tradicionales era significativa, por ello, debía explorarse la contribución de elementos hasta entonces omitidos, como las economías de escala, el progreso tecnológico y el mejoramiento en la calidad de la fuerza laboral. En particular, Denison señaló que el $23 \%$ del crecimiento anual del PNB norteamericano durante el periodo 1930-1960 se había potenciado por la mejora en el nivel educativo de la fuerza laboral de ese país. Este resultado, junto con el trabajo de Gary Becker (1964), formalizó la idea de “capital humano” y generó un campo de investigación en torno a la relación entre la economía y la educación. A partir de entonces se han hecho importantes contribuciones al diseño de la política económica y educativa. (Terrones, 1993)

Por otra parte, el nuevo contexto internacional exige que los países y sus sociedades se preparen para enfrentar desafíos de distinto orden: procesos de apertura económica, cambios constantes y crisis económicas. En tanto, la educación se percibe como una fuerza necesaria para la adaptación a la economía globalizada, el restablecimiento de la cohesión y la integración social; así como el alcance de metas democráticas a largo plazo y el aumento de las posibilidades de equidad y reducción de la pobreza para el desarrollo de los individuos, a nivel familiar y social. (Mosquera, 2010)

De esta manera, una persona que tiene acceso a la educación, posee más posibilidades de encontrar un trabajo e integrarse directamente al mercado laboral. 
Además, los países necesitan de personas con un nivel de educación alto para fomentar una economía basada en el conocimiento. Es esencial que los países aumenten su capacidad intelectual y eleven sus ingresos mediante el establecimiento de actividades productivas que requieran un nivel superior de educación y de aplicación científica. (Zottele \& Santiago, 2017)

Por otra parte, los hogares de menores ingresos cuentan con menos años de educación formal respecto a los "no vulnerables". A su vez, los "hogares pobres y vulnerables a la pobreza" de los países de menor desarrollo, presentan niveles muy bajos de logro educativo; esto explica por qué, a pesar de mostrar en algunos casos tasas de ocupación altas, no logran escapar de la pobreza o de la vulnerabilidad. Por tanto, la desigualdad en la dotación de capacidades humanas plantea, en algunas naciones, un serio obstáculo para lograr, en el futuro cercano, mejoras respecto a equidad social, por la vía de la reversión de la reproducción intergeneracional de la pobreza. (CEPAL, 2010)

Como afirma Chen (1991), una de las funciones de la educación es generar diversos tipos de trabajadores calificados que contribuyan al desarrollo de la sociedad. Según este pensamiento, la mejora de la capacitación de los trabajadores promueve aún más el desarrollo de la tecnología y de la administración, que constituyen un círculo de promoción mutua. (Ver Cuadro 1)

\section{Cuadro 1. Interrelación entre educación y productividad}

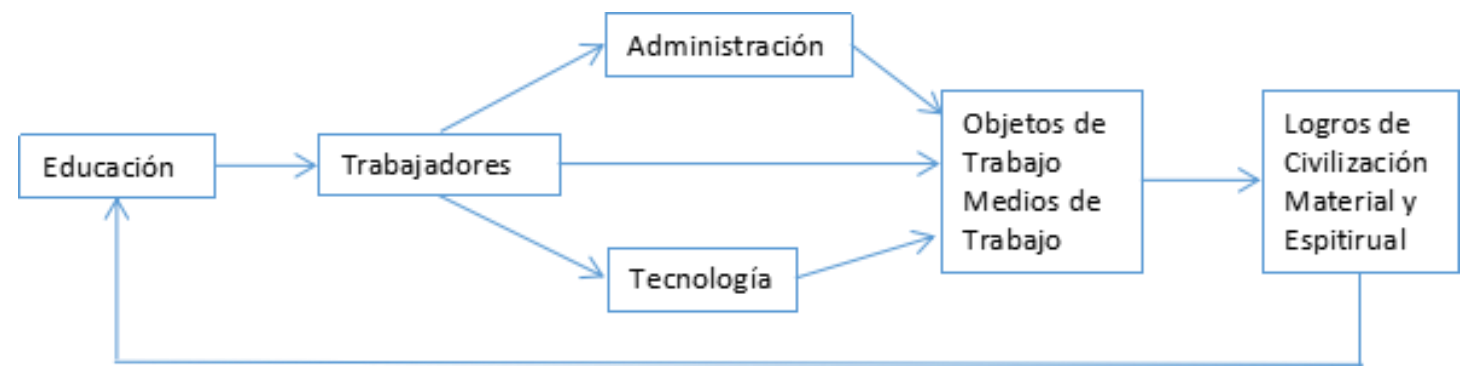

Fuente: Chen (1991)

La combinación orgánica de los cinco aspectos anteriores constituye el poder real de la productividad. A través de los factores humanos -trabajadores-y de los factores físicos -objeto de trabajo, medios del trabajo- se crea riqueza material y 
espiritual en el circuito de producción social. Por otra parte, el aumento en la riqueza social es correspondiente al incremento en la inversión en educación. Este es un círculo virtuoso entre el desarrollo educativo y la productividad.

En el mismo sentido, Gomez-Pinto (2014) señala que el proceso educativo puede brindar las condiciones necesarias para reducir las tasas de mortalidad de mujeres, niños y jóvenes, al incrementar las capacidades. De igual forma, Amartya Sen, premio Nobel de Economía de 1998, apunta que esta es una de las consecuencias directas, si la educación ingresa a los mercados como mecanismo para alcanzar la felicidad.

\subsection{La educación, un círculo virtuoso}

Cuando se habla de calidad de vida, la educación se puede citar en las dos puntas de la ecuación, ya sea como factor generador de mejora en la calidad de vida o como resultado de dicha mejora. En ese sentido, se puede analizar como un círculo virtuoso en el que mejores sistemas educativos y más abarcativos generarán mayores herramientas individuales y sociales para alcanzar una mejor calidad de vida; dicha mejora generará a su vez sistemas educativos más modernos. En tanto, el desarrollo social, económico y tecnológico, así como la mejora en la calidad de vida, van de la mano y no pueden ser considerados de manera individual, sino a través de una relación de interdependencia donde el progreso de uno signifique el progreso del otro. (Magallanes, 2012)

La calidad de vida de los pueblos y sus niveles de desarrollo económico están íntimamente asociados a la solidez de sus instituciones y a la calidad de su educación. Los países más desarrollados son aquellos que tienen las instituciones más sólidas; mientras que los de mejor calidad de vida tienen los mejores niveles de educación.

Por tanto, los economistas asignan una gran relevancia a la calidad del capital humano, al evaluar los factores que contribuyen al desarrollo. Esta calidad está determinada por el conocimiento, la creatividad, el esfuerzo, el trabajo en equipo 
y el cumplimiento de las normas de convivencia. La mayoría de estos factores son resultado de la educación, en el sentido más amplio de la palabra. (Clarín, 2005)

\section{Educación y mejora en la calidad de vida en China}

El 15 de abril de 1985, durante una reunión con el presidente de Tanzania, Ali Hassan Mwinyi, Deng Xiaoping expresó: "El sistema socialista que hemos establecido es un buen sistema y debe ser respetado. Ahora que estamos comprometidos en reformas económicas, todavía debemos adherirnos al camino socialista y a los elevados ideales del comunismo”. La generación más joven debe entender esta concepción sobre la historia de su país, pero la pregunta central es ¿qué es el socialismo y cómo se construye? Sobre esta temática, Deng dijo: "La pobreza no es socialismo, el socialismo debe eliminar la pobreza. Si no desarrollamos fuerzas productivas y no mejoramos el nivel de vida de la gente, no podemos decir que esté en línea con los requisitos socialistas". ${ }^{2}$

Qué es el socialismo y cómo construirlo, es una cuestión teórica importante; sin embargo, durante un largo periodo, este problema no ha sido dilucidado. Para entenderlo, es fundamental reconocer la esencia del socialismo. De acuerdo con los principios básicos del marxismo y la práctica del socialismo, Deng Xiaoping realizó incesantes exploraciones sobre este tema y, después de reflexionar profundamente, desarrolló una respuesta científica.

El líder enfatizó repetidamente que "la pobreza no es el socialismo"; según su visión, "la esencia del socialismo es liberar las fuerzas productivas, desarrollar las fuerzas productivas, eliminar la explotación, eliminar la polarización y, en última instancia, lograr la prosperidad común". Por lo tanto, las características del socialismo no son la pobreza, sino la riqueza, pero esta riqueza es la prosperidad común de la gente.

China mantiene un crecimiento estable de PIB, con un incremento anual de más del 6\% en los últimos años; en ocasiones llegando al 10\%, con un descenso notorio

\footnotetext{
2 Traducción propia.
} 
en el coeficiente de Gini y mejora en los índices del Programa de las Naciones Unidas para el Desarrollo (PNUD). Esta realidad marca una tendencia positiva, no solo en el crecimiento económico, sino también en algunos de los factores que promueven el desarrollo económico.

El coeficiente Gini en China presenta una tendencia decreciente, pero con una gran diferencia entre los datos según las regiones, lo que muestra que posee grandes diferencias en los índices de calidad de vida entre regiones, especialmente entre el Este y el Oeste. Dicha situación se refleja también en los sistemas educativos, ya que las universidades con los niveles más altos se encuentran en su mayoría en el Este del país.

En cuanto al IDH, China tiene el nivel más bajo, pero teniendo en cuenta el desarrollo desequilibrado entre las regiones, se destacan los índices de Beijing, Tianjin y Shanghái para el año 2016, que son $0.869,0.843$ y 0.852, respectivamente. Mientras que los de Guizhou, Yunnan-Tíbet-son los más bajos: 0.673, 0.668 y 0.6. (PNUD, 2016)

Desde la reforma y apertura en 1978, China ha promovido vigorosamente la educación obligatoria de nueve años, en un modelo de desarrollo educativo extenso, y ha intentado fomentar la expansión de la educación superior. A pesar de que la inversión en educación ha aumentado año tras año, la financiación para este aspecto sigue siendo relativamente insuficiente, obstaculizando el desarrollo de la misma.

En la década de 1990, Deng Xiaoping presentó la política nacional básica de "vigorizar el país a través de la ciencia y la educación”. Posteriormente, en 1993, propuso alcanzar el objetivo del 4\% del PIB para la educación fiscal nacional, pero el cronograma se retrasó hasta 2012. 


\section{Gráfica 1. PIB en valores actuales de dólar, 1970- 2014}

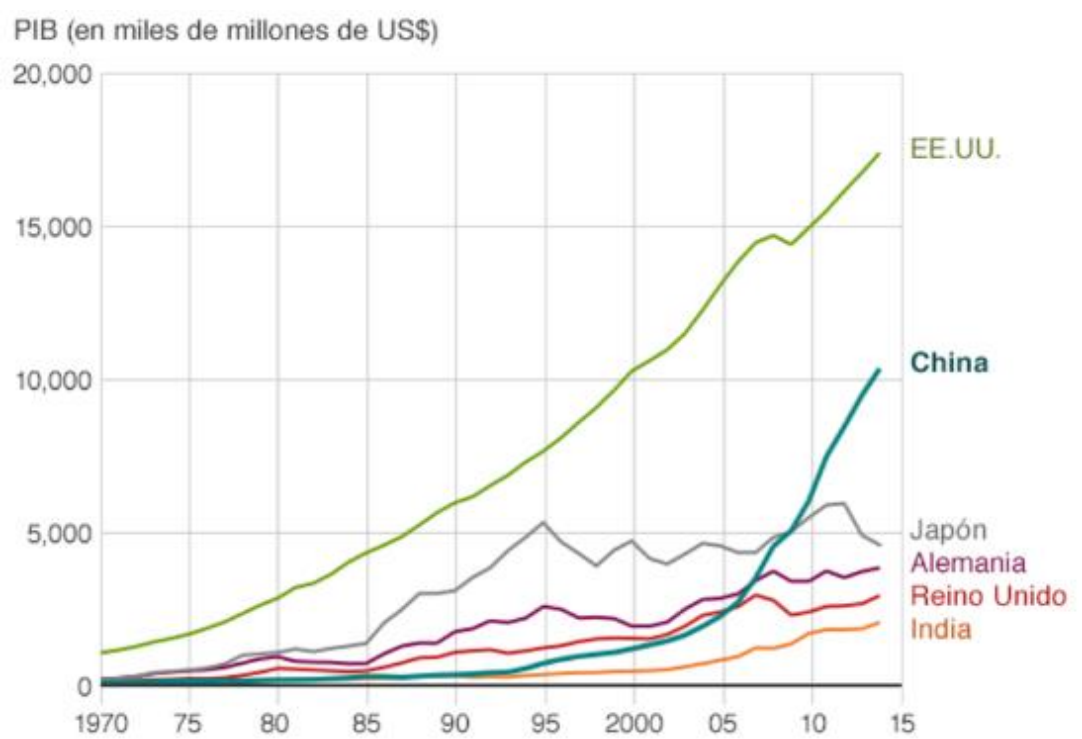

Funte: Banco Mundial.

En la Gráfica 1, se puede ver que, durante la última década, el aumento del PIB se acompaña de un incremento en el financiamiento para la educación. La financiación de la educación fiscal es dominante y representa el 4\% del PIB durante cinco años consecutivos a partir de 2012.

Como afirma Xiao (2017), desde el "XVIII Congreso Nacional del Partido Comunista de China", a través del apoyo y la orientación de fondos de educación financiera y políticas impositivas, la educación de China se ha desarrollado de manera integral. Por otra parte, la educación en las regiones centrales, en el oeste y en las áreas rurales se ha fortalecido. 


\section{Gráfica 2. Crecimiento de PIB y procentaje de gasto público en educación de China}

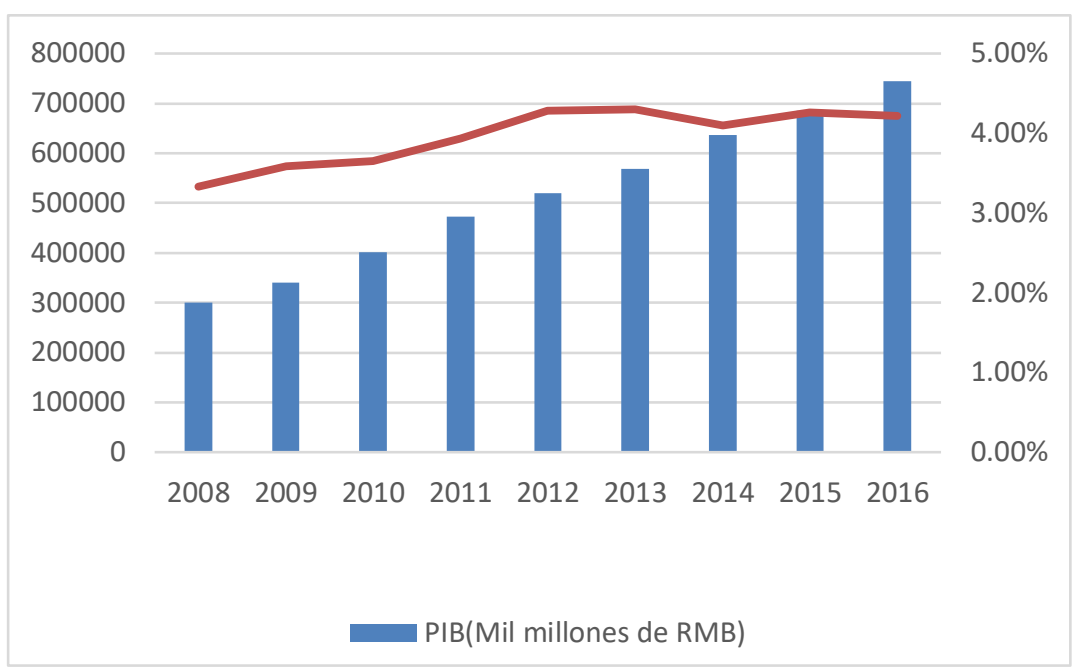

Fuente: Elaboración propia con base en los datos del 2008 al 2016 del Anuncio de estadísticas de implementación, del Fondo Nacional de Educación de Ministerio de Educación.

El presupuesto educativo de China mantiene una tendencia creciente durante los últimos años; es un gasto elevado en términos comparativos, considerando el PIB. En Estados Unidos y Alemania, el gasto público en educación representó el 4,9\% del PIB; mientras que Gran Bretaña y Francia y otros países superaron el 5,5\%, y Dinamarca alcanzó el 8,6\%. Por el contrario, todavía hay margen de mejora en la inversión de China en educación. (China report, 2017)

En tanto, el “Gaokao” (高考) es el examen nacional de acceso a la universidad. Desde su restablecimiento en 1977, tras la década de la Revolución Cultural, ha sido siempre escenario de una fuerte competición a nivel nacional. En 1977, un total de 5,7 millones compitieron por el acceso a 270,000 plazas. Tras años de reformas universitarias, en el año 2012 se contabilizó que 9,15 millones de estudiantes realizaron el examen, de los que un 75\% fueron aceptados (comparados con una tasa de aceptación del 56\%, en 1999). (Sánchez, 2013)

Según el vicepresidente ejecutivo de la Sociedad de Educación de China, Dai Jiagan (2017), restaurar el examen de ingreso a la universidad no implicó solo la restauración de un sistema educativo, sino también marcó el término de la lucha 
de clases y el inicio de la construcción económica, despertando los valores de la educación, el respeto por el conocimiento y el respeto por los talentos en toda la sociedad. Desde entonces, se abrió la etapa de construcción de un país fuerte de recursos humanos y se formó un mecanismo para la movilidad ascendente de los talentos, sentando las bases para la equidad y la justicia en la sociedad.

\section{Gráfica 3. Número de nuevos estudiantes universitarios (10,000 personas)}

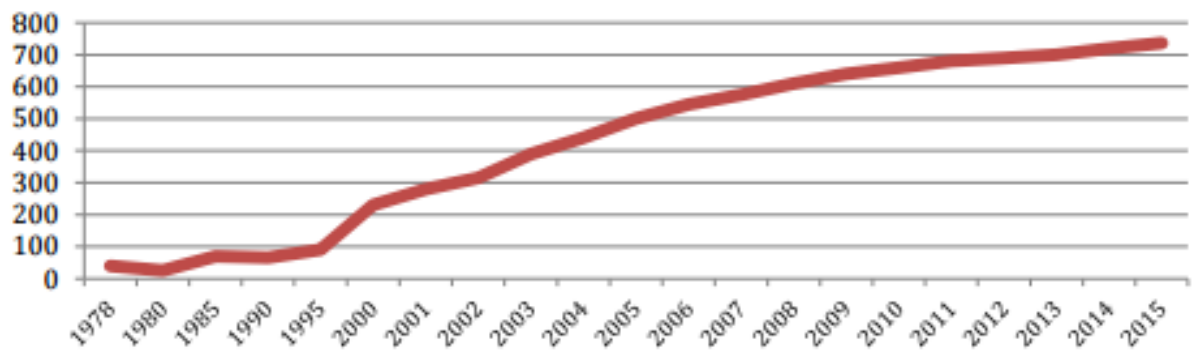

Fuente: Sánchez, 2013.

El siguiente gráfico muestra la relación entre los estudiantes matriculados en los distintos niveles educativos a lo largo de diez años. Mientras que, en 1998, un $65,90 \%$ de los estudiantes se encontraban en escuelas primarias y un $29,76 \%$ en escuelas secundarias, en 2011, el total de estudiantes en escuelas primarias fue del $48,47 \%$ y en secundarias, el $36,7 \%$.

Por otra parte, la educación superior es el nivel educativo que ha experimentado un crecimiento continuo a lo largo de la década analizada; esta tendencia ya se había dado en los años previos. No obstante, el crecimiento se ha ido ralentizando en el último periodo.

Es importante señalar que, aunque el número de alumnos que reciben educación superior ha aumentado año tras año, el porcentaje sobre el total de alumnos alcanzó, en 2011, solamente un poco más del 11\% del total de estudiantes; mientras que en el año 2000 llegó a un 2,59\%. (Sánchez, 2013) 
Gráfica 4. Porcentaje de estudiantes según nivel educativo

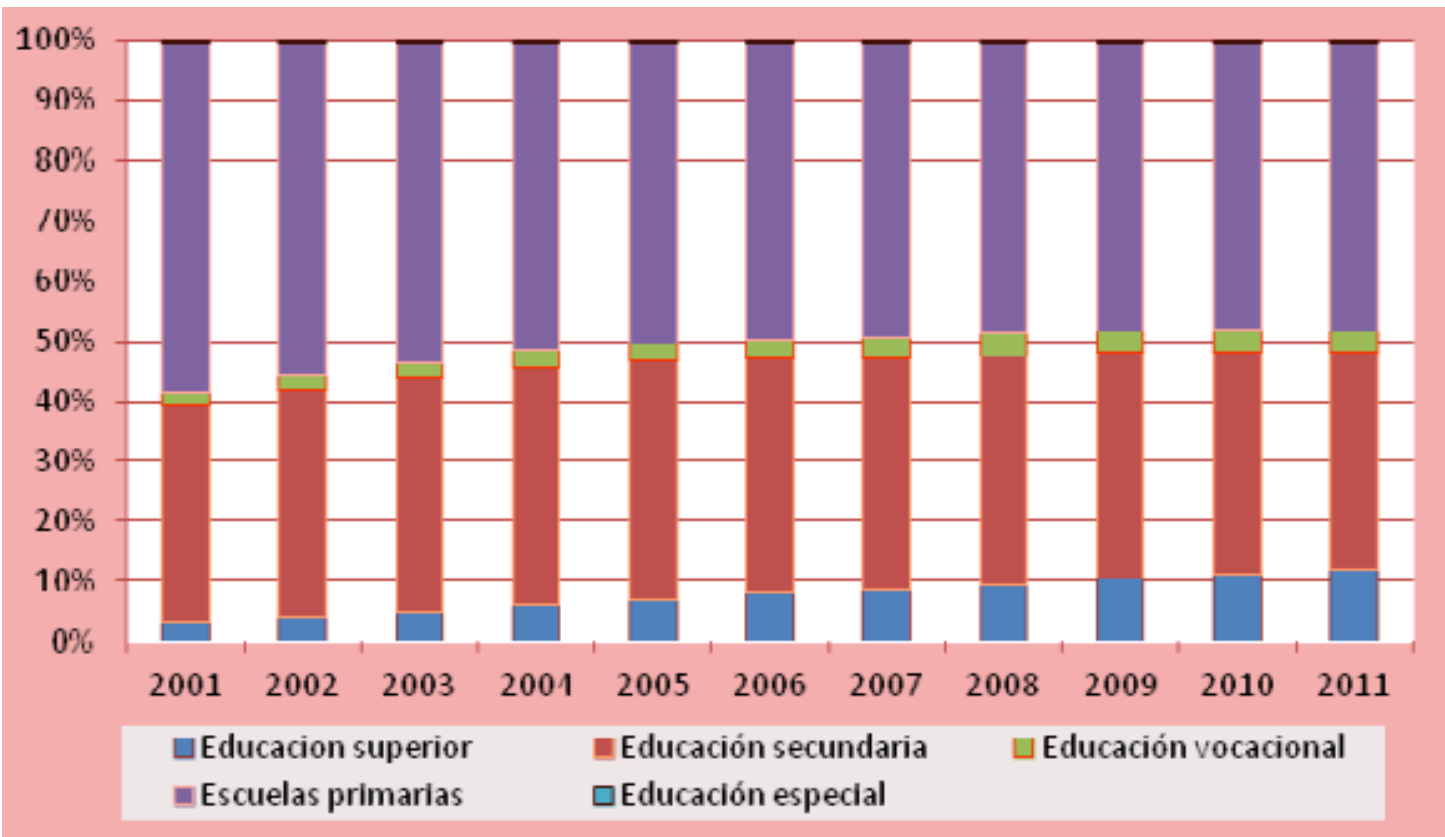

Fuente: Sánchez, 2013.

Se debe destacar que el número de estudiantes chinos en el extranjero ha ido aumentando progresivamente. El aumento ha sido mayor desde el año 2000, cuando un total de 340,228 estudiantes chinos cursaron programas en el extranjero, mientras que, a finales de 2011, la cifra fue de 1,985,545. (Sánchez, 2013).

\section{Gráfica 5. Número de estudiantes chinos en el extranjero del año 1978 al 2011}

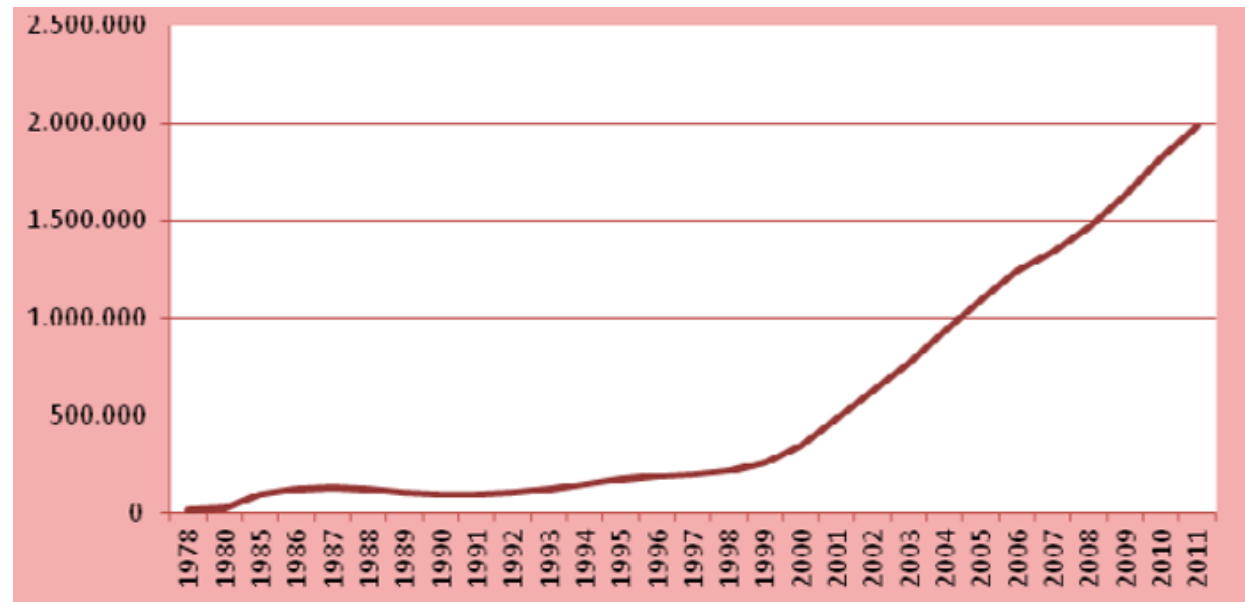

Fuente: Sánchez, 2013. 
China posee un desarrollo económico y una infraestructura educativa muy diferente en cada región administrativa; por lo que el nivel de educación per cápita es disímil. Por tanto, existe un desarrollo económico desequilibrado.

En esta sección, se analizarán las regiones de China continental, organizadas en 22 provincias, cinco territorios autónomos, cuatro ciudades autónomas (en total 31) y tres regiones: Este, Centro y Oeste. Esta clasificación se determinó de acuerdo con los tres cinturones económicos tradicionales del país, con el objetivo de explorar la relación entre educación y desarrollo económico en las tres regiones principales.

A continuación, se exponen gráficos del presupuesto educativo per cápita, del PIB per cápita y de la relación entre estos en el año 2008 y en el año 2016. Los datos contemplados provienen de 31 regiones chinas, de acuerdo con el Anuario estadístico de China, que incluye específicamente fondos de educación, PIB regional en cada provincia y población total del distrito. Dado que las estadísticas en varias regiones tienen un periodo de retraso de dos años, los datos retomados corresponden a los años 2008 y 2016. 


\section{Gráfica 6. Presupuesto de educación per cápita en 2008 y 2016 para 31} provincias chinas

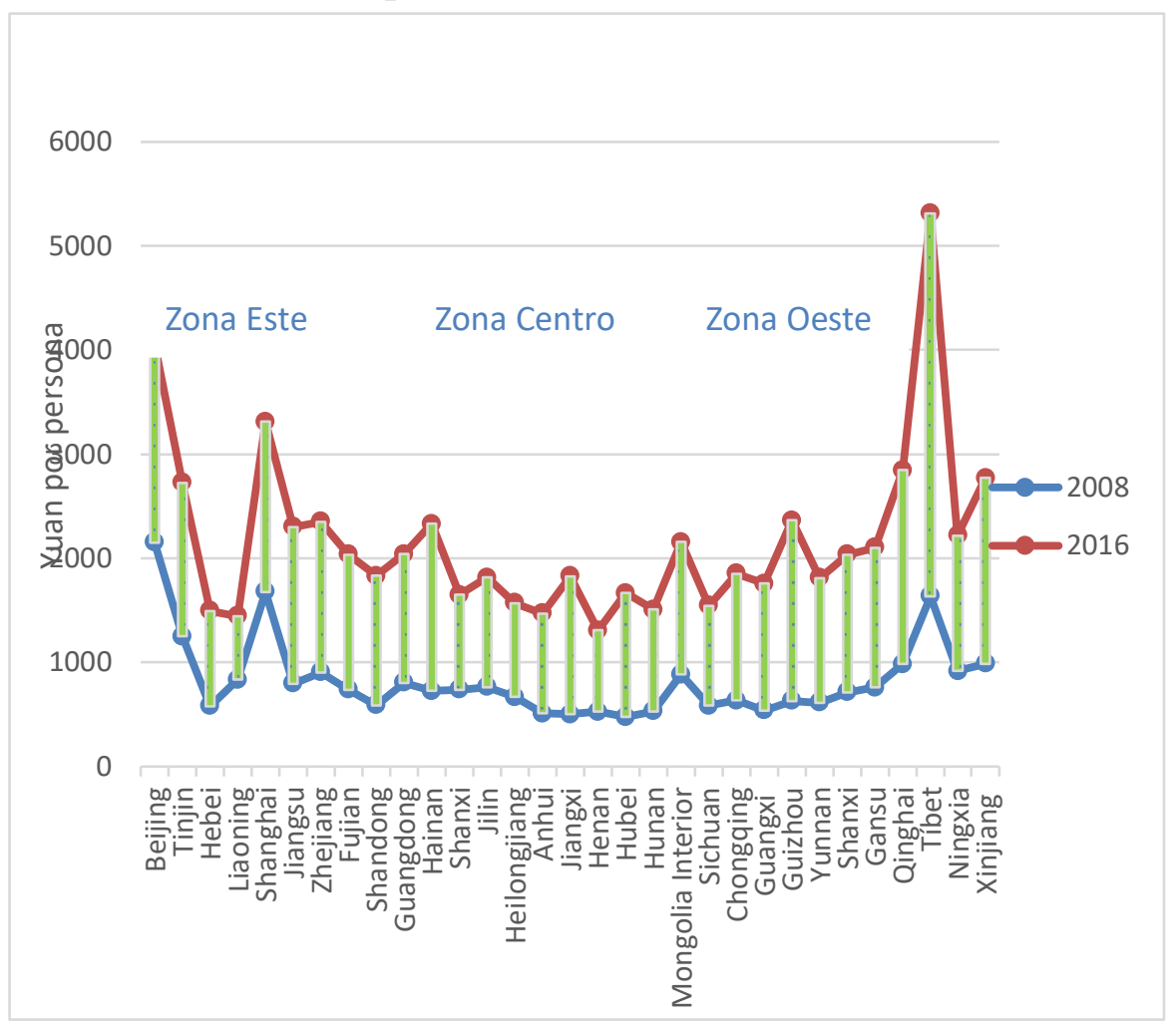

Fuente: Elaboración propia con base en los datos del Anuncio de estadísticas.

En la Gráfica 6 se comparan los datos de los años 2008 y 2016. Se puede observar un aumento significativo en financiación per cápita de educación de todas las provincias de China, pero las provincias no están equilibradas. La financiación de la educación per cápita en la región Este es generalmente elevada y en la mayoría de las provincias se ha duplicado la cifra. Los tres municipios de control directo de Shanghái, Tianjin y Beijing, mantienen el nivel más alto.

Por otra parte, la financiación de la educación per cápita de todas las provincias de la región central, se encuentra en un nivel relativamente bajo, tanto para el año 2008 como para el año 2016. En las provincias del Oeste es relativamente pequeña en 2008, pero la tasa de crecimiento de 2016 es notoria, especialmente para el Tíbet, Mongolia interior y la provincia de Qinghai, que son las más destacadas.

Sin embargo, considerando el método tradicional de dividir los tres cinturones económicos principales, se observa una gran diferencia entre las 
distintas provincias de la misma región. Los niveles de financiación de la educación per cápita en las provincias de Hebei y Shandong de la región Este son similares a los de la región Central, y apenas llegan a la mitad de Beijing y Shanghái.

Merece la pena mencionar la financiación de la educación per cápita en Mongolia interior, Tíbet, Ningxia y Xinjiang en la región Oeste, pues supera la de otras provincias e iguala la de algunas provincias del Este -Fujian, Zhejiang, Jiangsu y Liaoning.

Cabe aclarar que estas cuatro regiones corresponden a áreas donde habitan grupos minoritarios de China. En China existen 56 etnias, de las cuales 55 son minorías y la restante es la mayoría Han, con casi el 93\% de la población.

Gráfica 7. PIB per cápita por regiones en los años 2008, 2012 y 2018

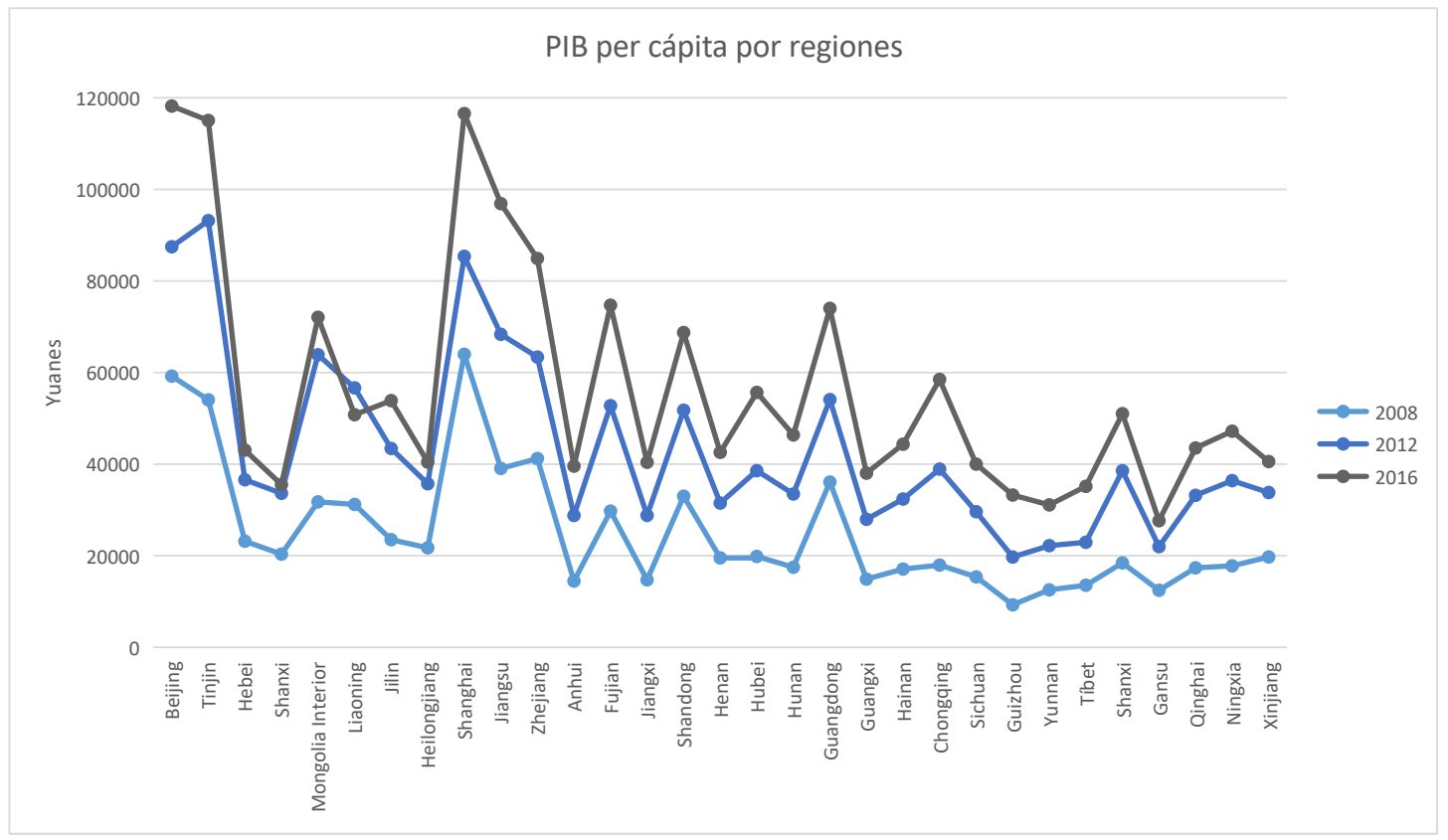

Fuente: Elaboración propia con base en los datos del Anuncio de estadísticas, del año 2008 al 2016

\section{Desafíos para el Sistema educativo chino}

\subsection{Popularización de la educación}

Se observa la necesidad de popularizar la educación obligatoria de nueve años, que desempeña un papel crucial en la mejora de la calidad de la población y el 
rápido desarrollo económico de las regiones del Centro y del Oeste de China. Ya que la mejora del nivel de educación implica un proceso largo, la inversión en educación en ambas regiones no debe poner demasiado énfasis en los beneficios a corto plazo. El Estado debe fortalecer los esfuerzos administrativos para implementar políticas educativas y complementarlas con las políticas preferenciales correspondientes.

\section{Gráfica 8. Reducción de la pobreza en China}

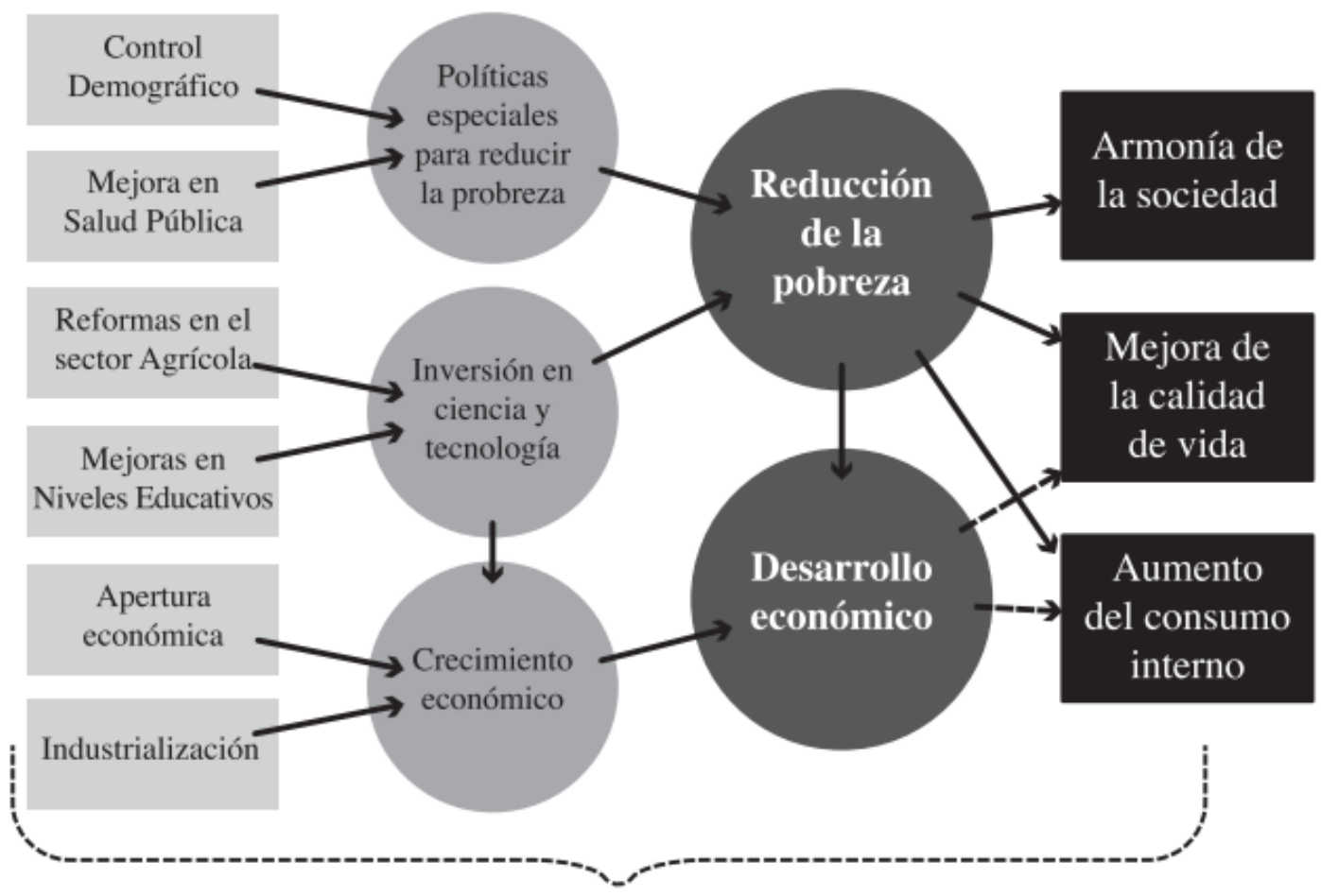

Socialismo del mercado

Fuente: Zottele, 2010.

En esta gráfica se puede apreciar que la mejora en los niveles educativos promueve en cierta medida el desarrollo económico. Las grandes transformaciones sólo han sido posibles gracias a la importancia que el gobierno ha otorgado a la educación y al desarrollo científico-tecnológico.

\subsection{Cross cultural basado en "La Franja y la Ruta"}

Actualmente, el gobierno chino promueve la Iniciativa de "La Franja y la Ruta" 
como una de sus políticas centrales de relaciones internacionales. Esta Iniciativa, que actualmente incluye a más de 70 países de diversas orientaciones políticas, religiones y con grandes diferencias culturales, requerirá de una mayor formación de estudiantes especializados en lengua, cultura, economía e historia internacional. Para ello, el sisema educativo chino deberá contemplar innovaciones que permitan incorporar todos elementos en los distintos niveles educativos, haciendo énfasis en la formación de los aspectos que generen líderazgo. Asimismo, en las Universidades se deberan generar especialidades relacionadas con "La Franja y la Ruta".

\subsection{Educación inclusiva: igualdad entre educación pública y privada}

Si se toma en cuenta la época posterior al año 1993, especialmente después de la promulgación de la "Ley de Promoción de la Educación Privada", de 2002, el sector privado ha experimentado un rápido desarrollo. De acuerdo con las estadísticas de educación publicadas por el Ministerio de Educación, en 20132015, el número de escuelas privadas chinas y el número de estudiantes inscritos está aumentando. En 2016 se registraron 171,000 escuelas privadas de varios tipos en todos los niveles, un aumento de 8,253 respecto al año anterior (2015), en el que se censaron 4,825,700 estudiantes; mismos que representaron un aumento de 2,539,500 respecto al año 2014. (Educación Sohu, 2016)

En tanto, en la zona Este de China hay más escuelas primarias y secundarias privadas; mientras que el número de estudiantes en las secundarias privadas de la región Central es elevado. Sin embargo, en la región Oeste, los estudiantes de escuelas secundarias son escasos, independientemente de la etapa de educación obligatoria o la etapa de la escuela secundaria.

Se puede observar que la educación primaria y secundaria privada en la zona de Este de China mantiene el desarrollo más fuerte; por otro lado, representa el más débil en el Oeste. Las escuelas primarias y secundarias privadas son servicios educativos diferenciados y su nivel de desarrollo está directamente relacionado 
con el nivel de desarrollo económico y social local.

Algunas famiilias de élite en las áreas desarrolladas de la Costa Sureste no están satisfechas con la educación de los exámenes competitivos nacionales y prefieren que sus hijos se inscriban en las escuelas primarias o secundarias internacionales privadas y se preparen para continuar sus estudios en el extranjero.

\subsection{Desigualdad entre regiones}

Como se ha mencionado, la infraestructura educativa en cada región administrativa es diferente y el nivel de educación per cápita es inconsistente. Generalmente, las zonas desarrolladas disponen de mejores recursos educativos que las zonas menos desarrolladas; las ciudades disponen de mejores recursos educativos que las zonas rurales.

Tabla 1. Número de las universidades de Proyecto 985 y 211, según regiones

\begin{tabular}{|c|c|c|}
\hline & $\begin{array}{c}\text { Universidades de } \\
\text { Proyecto 985 }\end{array}$ & $\begin{array}{c}\text { Universidades de } \\
\text { Proyecto 211 }\end{array}$ \\
\hline Zona Este & 24 & 68 \\
\hline Zona & 8 & 25 \\
Centro & 7 & 23 \\
\hline $\begin{array}{l}\text { Zona } \\
\text { Oeste }\end{array}$ & & \\
\hline
\end{tabular}

Fuente: Peng \& Chen, 2014.

El Proyecto 985 (en chino 工 程) fue anunciado en el año 1998, para promover el desarrollo y la reputación del sistema universitario chino. Las universidades patrocinadas por este proyecto durante el año 2014 fueron 31 .

Por otra parte, el Proyecto 211 (en chino 工程) inició en 1995 a cargo del Ministerio de Educación, con la intención de impulsar los niveles de investigación de las mejores universidades y cultivar estrategias de desarrollo socio-económico. Estos dos tipos de universidades son las mejores universidades 
en China y se puede observar que la zona Este se ubica la mayoría.

En 2013, The Rural Education Action Program (REAP) llevó a cabo ocho encuestas a gran escala, siguiendo la experiencia educativa de 20,000 niños en áreas rurales afectadas por la pobreza. Este programa encontró que la brecha entre la educación urbana y rural es muy grande: solo el 37\% de las personas en áreas rurales pobres completan el aprendizaje de la escuela secundaria, mientras que en la ciudad más del 90\% de estudiantes pueden terminar dichos estudios.

En este sentido, el equipo de profesores rurales y el diseño de la estructura disciplinaria deben mejorarse. Los profesoreses rurales a veces son mayores y la mayoría de ellos solo tiene nivel de educación secundaria; los profesores con altos niveles no quieren quedarse en las zonas rurales y poco desarrolladas.

De acuerdo con los resultados de la prueba del Banco Mundial sobre la educación estandarizada (TIMSS), en el año 2005, los niños rurales de cuarto grado solo pudieron alcanzar el nivel de educación estándar del tercer grado; mientras que los niños urbanos del mismo grado pudieron alcanzar el estándar del quinto grado. Esto demuestra que, en el cuarto grado, los estándares educativos de los niños urbanos y rurales fueron diferentes durante dos años; también puede explicar por qué hay cada vez menos niños de procedencia rural en la universidad.

Por otra parte, en el mes de mayo de 2010, el Consejo de Estado aprobó el "Plan nacional de reforma y desarrollo educativo a mediano y largo plazo (20102020)", cuyo objetivo principal es garantizar la igualdad en todo el sistema educativo del país. Uno de los principales problemas que hoy en día afectan la educación en China es el desequilibrado desarrollo entre áreas urbanas y rurales, por lo que el plan supone que se destinarán fondos para crear servicios de educación pública de estándar nacional en las zonas más desfavorecidas. El plan también recoge las modificaciones del sistema de entrada a las universidades, así como incentivos para que instituciones educativas privadas desarrollen un mayor papel en el sistema educativo. 


\subsection{Educación para las próximas generaciones}

El creciente número de estudiantes chinos que continúan su educación superior en el extranjero está determinado por diversos factores: mayor facilidad para viajar al exterior, la competencia de universidades extranjeras por captar estudiantes chinos, menos requisitos para acceder a muchas universidades extranjeras en comparación con las pruebas de selectividad locales, el valor añadido para el CV o el incremento de la renta en China.

Otro factor que determina que muchos estudiantes con poder adquisitivo prefieran la educación en el exterior, son las críticas que han venido exponiéndose en los foros educativos nacionales sobre el tipo de educación impartida en China. El sistema tradicional chino se caracteriza por el aprendizaje mediante memorización, la repetición incontestable de las enseñanzas del profesor y la falta de debate y trabajo en equipo, lo que se traduce en la ausencia de creatividad en las aulas y en la dificultad para fomentar talentos en nuevas áreas del conocimiento. (Sánchez, 2013)

Jack Ma, fundador y presidente del Grupo Alibaba, afirma que actualmente nos encontramos en el desafío de la educación de reformar el sistema para las nuevas generaciones. Cuando se trata de inteligencia artificial, Ma se posiciona como realista, pero también como optimista. "La nueva tecnología destruirá muchos puestos de trabajo, pero también creará muchos puestos más ", dijo; "la pregunta es si estamos listos y si estamos calificados para estos". ${ }^{3}$

Mientras "tanto, no debería haber mucha ansiedad por una guerra entre humanos y máquinas", pues "las computadoras nunca tendrán dominio sobre la "sabiduría" o el "amor", dando al hombre una ventaja perpetua. Los seres humanos deben tener la confianza en sí mismos. El ser humano tiene la sabiduría, la máquina no"; expresó el empresario.

\footnotetext{
${ }^{3}$ Expansión. (21 de septiembre de 2017). "Dejemos de entrenar a nuestros hijos para empleos manufactureros dice Jack https://expansion.mx/tecnologia/2017/09/21/dejemos-de-entrenar-a-nuestros-hijos-para-empleosmanufactureros-dice-jack-ma
} 
El visionario asiático anima a las instituciones de docencia a que cambien sus métodos de enseñanza, ya que, por mucho que se exija a los alumnos en temas de cálculo o dibujo, “una máquina siempre lo hará mejor”. Por eso, la clave para prosperar en un mundo de inteligencia artificial, robótica y realidad virtual, es la imaginación. Por ello, “debemos enseñar a nuestros jóvenes a ser innovadores y creativos. De esta manera encontrarán salidas profesionales".

\section{Conclusiones e investigaciones futuras}

Un análisis temporal de los indicadores de calidad de vida, del índice PNUD, del coeficiente de Gini y del PIB per cápita, nos muestra una constante mejora en la ecomonía y la calidad de vida de la poblacion de China. El alto nivel de gasto en educación es un factor que contribuye a este desarrollo; aunque en un país con territorio inmenso como es China, se puede observar desigualdad económica entre regiones, que coincide con la desigualdad en el acceso a la educación.

Estos factores, además, responden a los niveles de urbanización en el Oeste de China, donde se encuentran las regiones menos urbanas y más alejadas, y la población tiene menos acceso a la educación. Pero este no es el único tipo de desigualdad; en una misma región puede haber diferencia salarial, que se relaciona con el nivel de educación, la disparidad de calidad entre los servicios educativos públicos y los privados, la desigualdad educativa regional, etc. Estos y otros factores son algunos de los desafíos que todavía debe confrontar la reforma educativa en esta nación.

En China ha habido un desarrollo económico y una mejora de los indicadores de calidad de vida. Aunque el gasto de educación no es el único factor que ha contribuido al desarrollo del sistema educativo, se puede ver que, durante la última década, el aumento de la PIB ha estado acompañado de un incremento en el financiamiento para la educación.

Desde el "XVIII Congreso Nacional del Partido Comunista de China", a través del apoyo y la orientación de fondos de educación financiera y políticas 
impositivas, y mediante esfuerzos concertados de todas las partes, la educación de China se ha desarrollado de manera integral, y la educación en las regiones del Centro, Oeste y en las áreas rurales se ha fortalecido.

Por otra parte, la estabilidad política permite la aplicación de planes a largo plazo. Dicha estabilidad y plan integral de las políticas de China, se refleja en los indices de calidad de vida. En este sentido, la tendencia en los últimos años es una línea creciente.

Existen diversas investigaciones que se pueden realizar para profundizar el análisis de este trabajo. Una de ellas es la relación entre gasto en educaciòn y efectividad de las políticas; otra es la posibilidad de mutuo aprendizaje entre regiones, y el rol de la educación superior para la generación de políticas que apunten al desarrollo económico.

\section{Bibliografía}

Becker, G. (1964). Human capital. New York: Columbia University Press.

Cecchini, S. \& Rodrigo, M. (2011). "Protección social inclusiva: una mirada integral, un enfoque de derechos". En Libro de CEPAL, Núm. 111. Santiago de Chile: CEPAL.

CEPAL. (2010). Estudio Económico de América Latina y el Caribe 2009-2010: Impacto distributivo de las políticas públicas. Recuperado de: https://repositorio.cepal.org/bitstream/handle/11362/1070/1/20092010_es.pdf

Chen, Jingpu. (1991). "El ciclo virtuoso de la inversión educativa en la economía nacional”. En revista Educación y Economía, Núm. 4. China: Universidad Normal de China Central.

China Report. (2017) Análisis del gasto y la proporción de educación en China en 2017 (年我国教育经费支出及占比分析).

Clarín. (2005). Mejor educación asegura mayor calidad de vida. Consultado en: 
http:/edant.clarin.com/diario/2005/07/31/opinion/o-03402.htm

Denison, E. F. (1962). The Sources of Economic Growth in the United States and the Alternatives Before Us. New York: Committee of Economic Development.

Dennis, R.; Williams, W.; Giangreco, M. \& Cloninger, Ch. (1994). "Calidad de vida como contexto para la planificación y evaluación de servicios para personas con discapacidad”. En Siglo Cero, pp. 25, 155.

Educación Sohu. (2016). Libro Blanco de la Industria de la Educación de China 2016 (中国教育行业白皮书).

Gomez-Pinto, L. R. (2014). La política en educación como determinante de desarrollo económico: el caso de la educación de mujeres y menores de edad como variable para la educación de las tasas de mortalidad. Bogotá: Universitas.

Hulme, D. \& A., Shepherd. (2003). "Chronic poverty and development policy: an introduction”. En: World Development, Núm. 3 (31), pp. 55-75.

Magallanes, F. (2012). Educacion y calidad de vida.

Mosquera, A. (2010). “La educación y su efecto en la formación de capital humano y en el desarrollo económico de los países”. En Apuntes del CENES, Núm. $5(30)$, pp. $45-59$

Peng Xian \& Chen Lizao. (2014). Viendo la equidad de la educación superior de China desde la distribución regional de las universidades del proyecto $985 y 211$ (从 985 和 211 大学的区域分布 看我国高等教育的公平 性),煤炭高等教育, Núm. 3(32).

PNUD. (2016). Informe nacional de desarrollo humano de China. Recuperado de: http://hdr.undp.org/sites/default/files/reports/2783/undpch_nhdr_2016_en .$p d f$

PNUD. (2016). Informe sobre Desarrollo Humano. Recuperado de: http:/hdr.undp.org/sites/default/files/HDR2016_SP_Overview_Web.pdf

Sachs, J. (2017). El fin de la pobreza. Editorial Debate.

Sánchez, Eduardo. (2013). El sector de la educación en la R.P. China 
Schultz, T. W. (1961). “Investment in Human Capital”. En The American Economic Review.

Terrones, C. (1993). “Educación, capital humano y crecimiento económico: el caso de América Latina”. En Revista Economía, No. 31, pp. 23-70.

Xiao Jie. (24 de diciembre del 2017). "El nivel general de educación en nuestro país ha ingresado al mundo". En Guangming Daily.

Zottele, E. (2010). “China: 30 años de reformas y disminución de la pobreza. Políticas especiales y base ideológica”. En Orientando, Núm. 2 (1). Xalapa: Universidad Veracruzana.

Zottele, A., Li, Yan \& Santiago, M. (2017). Las Pymes mexicanas y chinas ante el crecimiento acelerado de las relaciones. México: Universidad Veracruzana. 